\title{
Detection of a novel porcine parvovirus, PPV4, in chinese swine herds
}

Lv Huang ${ }^{1}$, Shao-Lun Zhai ${ }^{1,2}$, Andrew K Cheung ${ }^{3}$, Hong-Biao Zhang ${ }^{1,4}$, Jin-Xue Long ${ }^{1 *}$, Shi-Shan Yuan ${ }^{1 *}$

\begin{abstract}
To determine whether the novel porcine parvovirus type 4 (PPV4) recently reported in America is prevalent in China, a set of specific primers was designed and used for molecular survey of PPV4 among the clinical samples collected from various provinces of China between 2006 and 2010. The results showed that PPV4 is present in Chinese swine herds at a rate of 2.09\% (12/573) among the clinical samples examined and 0.76\% (1/132) among the samples taken from healthy animals. We also noted that PPV4 was not detected in samples taken prior to 2009. Analysis of the coding sequences showed that the Chinese and American PPV4 genome sequences are closely related with greater than $99 \%$ nucleotide sequence identity. Similar to a previous study, viral genomes in head-totail configuration of various lengths of the non-coding region were detected. Our findings confirmed that PPV4 is a unique recently discovered virus in pigs. Phylogenetically, PPV4 is most closely related to bovine parvovirus 2 (BPV2, which is not a Bocavirus and is not assigned to any Parvovirinae genus) and shares limited ORF1 (33.6\%) and ORF2 (24.5\%) amino acid identity. With respect to genome structure and organization, PPV4 encodes an ORF3 in the middle of the viral genome that resembles the Bocavirus genus. However, the PPV4 ORF3 encoded protein shares minimal amino acid identity with the ORF3 encoded proteins of the Bocavirus genus.
\end{abstract}

\section{Findings}

The International Committee on Taxonomy of Viruses has classified the Parvovirinae subfamily of viruses into five genera: Dependovirus, Bocavirus, Erythrovirus, Parvovirus and Amdovirus. Recently, two new genera, Hokovirus and Cnvirus, have been proposed. Hokovirus includes a group of porcine and bovine isolates that clustered with the human PARV4 and PARV5 viruses $[1,2]$ and Cnvirus includes the H-1 isolate from Myanmar [3] and a group of newly identified porcine isolates from China that exhibit a distinct sublineage from the Hokovirus [4,5]. In addition, there are two bovine isolates (BPV2 and BPV3) and one porcine isolate (PPV4) that do not cluster with any member of the Parvovirinae genera [6]. The genomes of the Parvovirinae members are linear single-stranded DNA of about 5 kilobases that contain terminal palindromic sequences. In general, the genome encodes two major open reading frames (ORFs) coding for the non-structural protein(s) located at the 5 '-end and the capsid protein(s) located at the 3'-end.

\footnotetext{
* Correspondence: longjx@shvri.ac.cn; shishanyuan@shvri.ac.cn 'Department of Swine Infectious Diseases, Shanghai Veterinary Research Institute, Chinese Academy of Agricultural Sciences, Shanghai 200241, China Full list of author information is available at the end of the article
}

An additional ORF3 located in the middle of the viral genome was observed among viruses of the Bocavirus genus (bovine parvovirus [BPV1], canine minute virus [MVC], human bocavirus [HBoV], and, potentially, a partially sequenced porcine boca-like virus [Pbo-likeV]) $[7,8]$. PPV4 is unique in that its genome nucleotide sequence is most related to BPV2, but the coding capacity and genome organization are more related to viruses of the Bocavirus genus [6]. Limited amino acid identity, ORF1 (33.6\%) and ORF2 (24.5\%), exists between PPV4 and BPV2. The ORF3 amino acid identity among the three recognized Bocavirus members is 43.347.0\%. Whereas the Pbo-likeV ORF3 shares 33.6-35.6\% amino acid identity with the recognized Bocaviruses, the PPV4 ORF3 shares only 4.9-11.2\% [6-8]. The ORF3 of Pbo-likeV and PPV4 share low nucleotide identity (45\%) and amino acid identity (17.5\%), which indicate PbolikeV and PPV4 are two distinct parvoviruses.

The aim of this study was to investigate whether PPV4 exists among swine herds in China by a PCR assay. A total of 705 animals were examined. A sample was collected from each pig (573 sick animals and 132 healthy animals) in 12 provinces of China from 2006 to 2010 (100 samples in 2006, 100 samples in 2007,100 samples
C Biomed Central

(c) 2010 Huang et al; licensee BioMed Central Ltd. This is an Open Access article distributed under the terms of the Creative Commons Attribution License (http://creativecommons.org/licenses/by/2.0), which permits unrestricted use, distribution, and reproduction in any medium, provided the original work is properly cited. 
in 2008,281 samples in 2009,124 samples in 2010). The sick pigs exhibited clinical symptoms that encompassed trembling, fever, testicular atrophy, abortion and death. The samples included 386 sera and 319 tissues from various organs. Briefly, viral DNA was extracted using a DNA/RNA extraction kit (catalog \# DP315, Tiangen Biotech Inc., Beijing, China). Based on the PPV4 genomic sequence (GenBank accession numbers: GQ387499) [9], a pair of detection primers [PPV4-F (5'GGGCGAGAACATTGAAGAGGT-3') and PPV4-R (5'TTGTGAGTATGGGTATTGTGT-3')] targeting a 543 nucleotide sequence within ORF2 were used. The PCR reaction was performed according to manufacturer's instruction (catalog \# KT201-2, Tiangen Biotech Inc., China). The cycling program was as follows: pre-denaturation at $95^{\circ} \mathrm{C}$ for $5 \mathrm{~min}, 40$ cycles of $95^{\circ} \mathrm{C}$ for $30 \mathrm{~s}$, $53^{\circ} \mathrm{C}$ for $30 \mathrm{~s}$ and $72^{\circ} \mathrm{C}$ for $40 \mathrm{~s}$, final extension for 10 min at $72^{\circ} \mathrm{C}$. The PCR products were gel-purified and cloned into the PCR2.1T/A vector (Invitrogen Corporation, California, U.S.A.). Nucleotide sequences were determined using an AB-3730 automated DNA sequencer and the results obtained were analyzed with the BLASTx program http://www.ncbi.nlm.nih.gov/ blast/. The results showed that 12 out of 573 clinical samples and 1 out of 132 healthy pig samples were positive for PPV4. PPV4-positive pigs were not detected among samples collected from 2006-2008. Eight of the positive samples were collected in 2009 and the other five were collected in 2010 and they came from five different breeding pig farms located in three provinces of China. Although the overall detection rate was low $(13 / 705=1.84 \%)$, the rate in each positive farm was high: $37 \%$ (7/19), $25 \%(1 / 4), 40 \%$ $(2 / 5), 20 \%(2 / 10)$ and $50 \%(1 / 2)$. Among the thirteen PPV4-positive samples identified, 10 samples were from adult pigs ( 7 sows about seven months and 3 boars about four months old), two from a dead piglet (about 8 weeks old) and one from a healthy piglet (about 4 weeks old). The adult pigs were diagnosed with reproductive failure, while the sick piglet had displayed fever and neurologic symptoms. Further analysis showed that PPV4 DNA existed in the heart, serum, lymph node, lung and kidney, with the heart containing the highest viral load (data not shown).

To explore whether there were any co-infecting viruses present in our PPV4-positive pigs, we investigated the presence of porcine reproductive and respiratory syndrome virus (PRRSV), porcine circovirus 2 (PCV2), classic swine fever (CSFV), PPV1, PPV2, PPV3, pseudorabies virus $(\mathrm{PrV})$ and porcine torque teno virus (genogroups 1 [PTTV1] and 2 [PTTV2]) according to the protocols previously described [1,3,9-11]. The results revealed that all 13 PPV4-positive samples were coinfected with both PTTV1 and PTTV2, while 4 out of
Table 1 Primers for the amplification of the full-length genome sequence of PPV4.

\begin{tabular}{|c|c|c|c|c|}
\hline$\#$ & Primers & Sequences & Position* & $\begin{array}{l}\mathrm{Tm} \\
\left({ }^{\circ} \mathrm{C}\right)\end{array}$ \\
\hline \multirow[t]{2}{*}{1} & PF356 & $5^{\prime}$ TGACGCAGTACAGACCGACGAGA $3^{\prime}$ & 356 & 60 \\
\hline & PR1197 & 5' AATGCAAGTGCAAGCCACCTTाT 3' & 1197 & \\
\hline \multirow[t]{2}{*}{2} & PF1050 & 5' AGTAATCTGGTAATCGCTGTTCG 3' & 1050 & \\
\hline & PR1942 & 5' ATGTTAGTCTTTCCTGTTGTGGC 3' & 1942 & \\
\hline \multirow[t]{2}{*}{3} & PF1568 & 5' GCTGGTGGATAACAACATCTGCT 3' & 1568 & \\
\hline & PR2553 & 5' GTTCTTCTTTCTCGGTGCTTCT 3' & 2553 & \\
\hline \multirow[t]{2}{*}{4} & PF2530 & $5^{\prime}$ AAGAAGCACCGAGAAAGAAGAAA 3' & 2530 & \\
\hline & PR3413 & 5' AAATCTAAGGGACAAGGCAAACG 3' & 3413 & \\
\hline \multirow[t]{2}{*}{5} & PF3363 & $\begin{array}{l}5^{\prime} \text { AGATACTAAGAAAGACAAGG } \\
\text { TGGAG 3' }\end{array}$ & 3363 & \\
\hline & PR4263 & $\begin{array}{l}5^{\prime} \text { AATAATAGAAGGTATAG } \\
\text { CGTCTCCA } 3^{\prime}\end{array}$ & 4263 & \\
\hline \multirow[t]{2}{*}{6} & PF3918 & 5' ACCTGCTCCTCCATCTTCTCCAC 3' & 3918 & \\
\hline & PR4897 & 5' GGCCGTCATCATACATTCTGCTC 3' & 4897 & \\
\hline \multirow[t]{2}{*}{7} & PF4219 & 5' ACTTACTGTTCTATGATGTCTGGAG 3' & 4219 & \\
\hline & PR5862 & 5' ATATCATCTGCGGTGTCTGGG 3' & 5862 & \\
\hline
\end{tabular}

*The position according to PPV4 clone C17 GenBank accession number: GQ387500) [6]

13 samples (30.7\%) were co-infected with PCV2. The other viruses examined were not detected.

Three PPV4-positive pigs (2 sows and 1 boar) from two farms in two provinces: Jiangsu (JS0910 and JS0918, from the same farm) and Henan (HEN0922) were selected for full-length genome sequence determination. Seven pairs of primers (Table 1) (based on of GenBank sequence GQ387500) [6] were designed and used to determine the coding sequences, in the 5' to 3 ' orientation, from the beginning of ORF1, through ORF3, to the end of ORF2. The nucleotide sequences obtained were assembled using the Seqman software and aligned using CLUSTALW of alignment software and a phylogenetic tree was generated using MEGA 4.1 software [12] and the neighbor-joining method [13].

Sequence analysis revealed that the JS0910 sample yielded 2 variants, the JS0918 sample yielded 3 variants and the HEN0922 sample yielded 2 variants. These seven PPV4 genomes contained three major ORFs, which included a putative replicase (ORF1), an ORF3 of unknown function and a capsid gene (ORF2). The coding capacity of each ORF was identical to that of the American PPV4 [6]. Phylogenetic analysis showed that these Chinese PPV4 genomes clustered with the American PPV4 genomes. Among the Chinese isolates, the nucleotide sequence identity of ORF1 was $99.1-99.3 \%$, ORF2 was 99.2-99.8\% and ORF3 was 99.2-99.3\%. Between the Chinese isolates and the American isolates, nucleotide identity of ORF1 was $99.7-99.8 \%$, ORF2 was 99.2-99.8\% and ORF3 was 99.7-99.8\%. Among the Chinese isolates, the amino acid identity of ORF1 was 
98.9-99.3\%, ORF2 was $99-100 \%$ and ORF3 was $99.3-$ 99.6\%. Between the Chinese isolates and the American isolates, the amino acid identity of ORF1 was $99.8-100 \%$, ORF2 was 98.5-99\% and ORF3 was 99.7-99.9\%.

Previous work demonstrated that head-to-tail circular or concatameric PPV4 genomes exist in tissues of the infected animals [6]. Primers designed specifically to detect head-to-tail covalently linked sequences used in the previous study were employed to determined the head-to-tail junction sequences of the seven PPV4 genomes obtained. PCR experiments yielded head-to-tail products of four difference sizes. After sequence determination and assembly, it was noted that the sizes of the seven genomes ranged from 5400 to5644 nucleotides (nt) (GenBank accession numbers: GU978964-GU978968, HM031134-HM031135). In comparison with the genomes of the American PPV4 (5780-5905 nt), all the Chinese PPV4 genomes examined were smaller and the deleted sequence in each genome was located between $\mathrm{nt}$ 5964-595 in the non-coding region. The $5400 \mathrm{nt}$ genome (GU978964, GU978968 and HM031134) was detected in all 3 pigs that came from 2 different provinces and the 5644 nt genome (GU978965 and GU978967) was detected in 2 pigs from the same farm. Interestingly, the nucleotide deletions of the three $5400 \mathrm{nt}$ genomes were identical and the nucleotide deletions of the two $5644 \mathrm{nt}$ genomes were also identical.

In conclusion, the results of this study demonstrated that PPV4 existed in some breeding pig farms in China, probably since 2009. Phylogenetically, the Chinese and American PPV4s are closely related and cluster in the same clade. They share greater than $99 \%$ nucleotide sequence identity in all 3 ORFs. The American PPV4 was first identified among swine suffering from an acute onset disease of high mortality in North Carolina, USA during late 2005 [14]. Examination of the farm records showed that the two PPV4-positive Chinese farms had purchased swine from a common source in North Carolina prior to the discovery of PPV4. Since the Chinese and American PPV4s are very similar with respect to nucleotide sequence and deletions in the non-coding region, it is possible that PPV4 was introduced into China through the importation of PPV4-infected pigs from the U.S. This study also confirmed previous study [6] that circular or head-to-tail concatameric PPV4 molecules were present in the the diseased animals. The head-to-tail configured PPV4 genomes are likely generated via circularization of the genome in which the left-end and right-end of the linear genome are covalently linked. It is not surprising that some terminal nucleotides of the linear genome may have been deleted during the circularization process, which is observed in this and previous studies. Similar to that of a Dependovirus, adeno-associated virus type 2 , the presence of head-to-tail configured molecules in the infected animals suggests that PPV4 may be able to establish persistence in its host $[15,16]$. The fact that PPV4 was detected in diseased animals with other co-infecting viruses, further investigations are needed to determine the pathogenic capability of PPV4 and surveillance studies are needed to determine whether PPV4 could spread and become a serious emerging swine pathogen.

\section{Acknowledgements}

The authors are grateful to Dr. Da-Wei Xu at Boerhinger Ingelheim Inc. and Xiang-Jian Li at China Animal Husbandry Inc. for providing the clinical samples. We thank Dr. Marcus Kehrli at USDA-NADC for critical review of the manuscript. This project was partially sponsored by a Basic Research Project grant from the National Nonprofit Research Institutions (2010BJ05) to J.L, and National Project funding from the Ministry of Science and Technology (MOST, 2006BAD06A01) and Shanghai Agricultural Research Fund (2006-10-4) to S.Y.

\section{Author details}

${ }^{1}$ Department of Swine Infectious Diseases, Shanghai Veterinary Research Institute, Chinese Academy of Agricultural Sciences, Shanghai 200241, China. ${ }^{2}$ College of Veterinary Medicine, Xinjiang Agricultural University, Urumqi 830052, China. ${ }^{3}$ Virus and Prion Research Unit, National Animal Disease Center, PO Box 70, Ames, IA 50010, USA. ${ }^{4}$ College of Veterinary Medicine, Nanjing Agricultural University, Nanjing 210095, China.

\section{Authors' contributions}

LH, SLZ and HBZ obtained samples and extracted viral DNA. LH carried out the PCR and sequencing studies and drafted the manuscript. JXL, AKC and SSY conceived the study and reviewed the manuscript. All authors read and approved the final manuscript.

\section{Competing interests}

The authors declare that they have no competing interests.

Received: 7 September 2010 Accepted: 21 November 2010 Published: 21 November 2010

\section{References}

1. Lau SK, Woo PC, Tse H, Fu CT, Au WK, Chen XC, Tsoi HW, Tsang TH, Chan JS, Tsang DN, et al: Identification of novel porcine and bovine parvoviruses closely related to human parvovirus 4. J Gen Virol 2008, 89:1840-1848.

2. Szelei J, Liu K, Li Y, Fernandes S, Tijssen P: Parvovirus 4-like virus in blood products. Emerg Infect Dis 16:561-564.

3. Hijikata M, Abe K, Win KM, Shimizu YK, Keicho N, Yoshikura H: Identification of new parvovirus DNA sequence in swine sera from Myanmar. Jpn $\rfloor$ Infect Dis 2001, 54:244-245.

4. Wang F, Wei Y, Zhu C, Huang X, Xu Y, Yu L, Yu X: Novel parvovirus sublineage in the family of Parvoviridae. Virus Genes 2010, 41(2):305-8.

5. Cheng W, Li J, Huang C, Yao D, Liu N, Cui S, Jin Y, Duan Z: Identification and Nearly Full-Length Genome Characterization of Novel Porcine Bocaviruses. PLOS ONE 2010, 5(10):e13583.

6. Cheung AK, Wu G, Wang D, Bayles DO, Lager KM, Vincent AL: Identification and molecular cloning of a novel porcine parvovirus. Arch Virol 2010, 155:801-806.

7. Blomström AL, Belák S, Fossum C, McKillen J, Allan GM, Wallgren P, Berg M: Detection of a novel porcine boca-like virus in the background of porcine circovirus type 2 induced postweaning multisystemic wasting syndrome. Virus Research 2009, 146:125-129.

8. Blomström AL, Belák s, Fossum C, Fuxlerc L, Wallgren P, Berg M: Studies of porcine circovirus type 2, porcine boca-like virus and torque teno virus indicate the presence of multiple viral infections in postweaning multisystemic wasting syndrome pigs. Virus Res 2010, 152(1-2):59-64. 
9. Li X, Xu Z, He Y, Yao Q, Zhang K, Jin M, Chen H, Qian P: Genome comparison of a novel classical swine fever virus isolated in China in 2004 with other CSFV strains. Virus Genes 2006, 33:133-142.

10. Yue F, Cui S, Zhang C, Yoon KJ: A multiplex PCR for rapid and simultaneous detection of porcine circovirus type 2, porcine parvovirus, porcine pseudorabies virus, and porcine reproductive and respiratory syndrome virus in clinical specimens. Virus Genes 2009, 38:392-397.

11. Taira O, Ogawa H, Nagao A, Tuchiya K, Nunoya T, Ueda S: Prevalence of swine Torque teno virus genogroups 1 and 2 in Japanese swine with suspected post-weaning multisystemic wasting syndrome and porcine respiratory disease complex. Vet Microbiol 2009, 139:347-350.

12. Tamura K, Dudley J, Nei M, Kumar S: MEGA4: Molecular Evolutionary Genetics Analysis (MEGA) software version 4.0. Mol Biol Evol 2007, 24:1596-1599.

13. Saitou N, Nei M: The neighbor-joining method: a new method for reconstructing phylogenetic trees. Mol Biol Evol 1987, 4:406-425.

14. Cheung AK, Lager KM, Kohutyuk Ol, Vincent AL, Henry SC, Baker RB, Rowland RR, Dunham AG: Detection of two porcine circovirus type 2 genotypic groups in United States swine herds. Arch Virol 2007, 152:1035-1044.

15. Schnepp BC, Jensen RL, Chen CL, Johnson PR, Clark KR: Characterization of adeno-associated virus genomes isolated from human tissues. J Virol 2005, 79:14793-14803.

16. Schnepp BC, Jensen RL, Clark KR, Johnson PR: Infectious molecular clones of adeno-associated virus isolated directly from human tissues. J Virol 2009, 83:1456-1464.

doi:10.1186/1743-422X-7-333

Cite this article as: Huang et al: Detection of a novel porcine parvovirus, PPV4, in chinese swine herds. Virology Journal 2010 7:333.

\section{Submit your next manuscript to BioMed Central} and take full advantage of:

- Convenient online submission

- Thorough peer review

- No space constraints or color figure charges

- Immediate publication on acceptance

- Inclusion in PubMed, CAS, Scopus and Google Scholar

- Research which is freely available for redistribution

Submit your manuscript at www.biomedcentral.com/submit
Ciomed Central 